\title{
An Approach for Reducing Gas Turbines Usage by Wind Power and Energy Storage
}

\author{
Nejm Saadallah ${ }^{1} \quad$ Yngve Heggelund $^{2}$ \\ ${ }^{1}$ Norwegian Research Centre, \{nsaa, ynhe $\}$ enorceresearch.no
}

\begin{abstract}
Offshore oil and gas platforms can accelerate their shift towards lower greenhouse gases emissions by combining wind power generation and energy storage systems. However, the variability of the wind resources and power demand, and the limited storage capacity make the design of the system particularly challenging. We present a modelica library at its early stage of the development and yet with promising results. The model will be used to simulate the dynamics of the whole system for a long period of time using simplified power components and balanced micro grid. This paper shares some preliminary results by applying the model on a dataset of wind resources and power demand from the North Sea. The results are the power system dynamics and an approximation of the mass of greenhouse gas emissions. On the long term the model will be applied to determine the right control and optimization strategy to control the energy system towards lower greenhouse gas emissions without compromising the balance between the power supply and demand.
\end{abstract}

Keywords: power systems, modelica, oil platform electrification

\section{Introduction}

Offshore wind resources in Norway were estimated to be close to 12000 terawatt hours per year as per 2018 (Bosch, Staffell, and Hawkes 2018). This great energy potential combined with the increasing offshore wind turbine's power generation offer good opportunities to reduce the amount of greenhouse gases. Emissions are mainly due to gas turbines power generation on offshore oil and gas platforms. Most of today's oil and gas platforms are powered using up to three gas turbines. This has shown to be a reliable solution but comes at the cost of important amounts of CO2 and NOx emissions (SSB 2020). In addition, introducing variable energy sources like wind power without energy buffers, combined with the fact that gas turbines take a significant time to get started means that they have to be kept at their idle state (ready to generate power) for long periods of time. Unfortunately, this leads to release of greenhouse gas emissions without even providing power. In this paper we explore the possibility to offload gas turbines by integrating wind turbines and energy storage systems. This paper builds on earlier work on integrating offshore wind farms with nearby oil plat- forms (He et al. 2010), by adding an energy storage capacity, and dynamical model based on energy balance. The objective is to provide a simple to configure and fast to run modelica model (ELOGOW 2021) that can be used to quantify the reduction of greenhouse gas emissions under realistic operational conditions over a long period. The case is given by a dataset for the power demand, and wind resources (ERA5 2020). The variability of the wind resources and the demand makes it challenging to keep the system in balance. Part of our approach is to exploit possible imbalance (deficit, or losses) to qualify a design composed of physical and control sub-systems. On the long terms, the model can be used to provide decision support based on the relation between wind resources, power generated, energy storage capacity, and control strategy to reduce greenhouse gas emissions. This model is intended to be generic in the sense where it does not require detailed component design.

\section{Model}

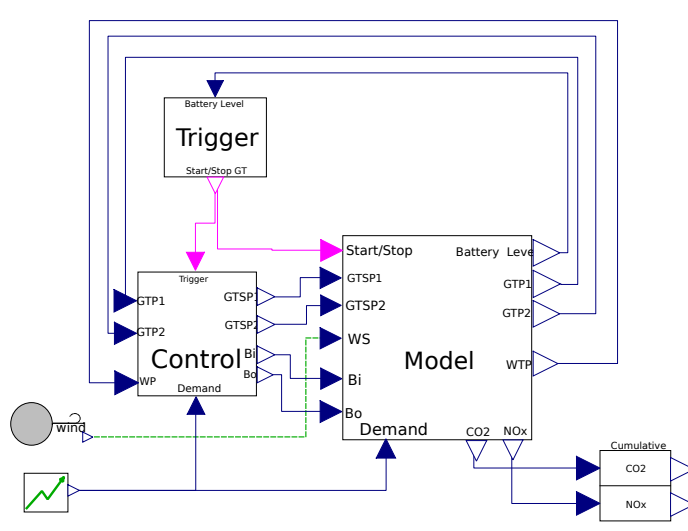

Figure 1. Simulation Overview

\subsection{Simualtion Overview}

Figure 1 shows the three main blocks used in the simulation. The "Model" block simulates the dynamic of the micro grid and all the components that are connected to it. This block will be further explained in the following sections. The control system strategy relies on the battery level provided by the "Model" block, and the setpoints to control the power supply by the gas turbines and the battery as well as the power for charging and draining the battery. The "Trigger" block defines two limits: a low limit 
and a high limit. When the low limit is reached $30 \%$ battery level in this paper), a signal is emitted to warmup the gas turbines, and the gas turbines' power setpoints are chosen in a way to balance the power demand, plus a supplementary power to recharge the battery. On the other hand, when the high limit is reached (60\% in this paper), the gas turbines are turned off, and the battery takes over the required power supply. Note that we use this approach for testing the model, and possible improvements will be discussed later in this paper.

\subsection{Physical Components Overview}

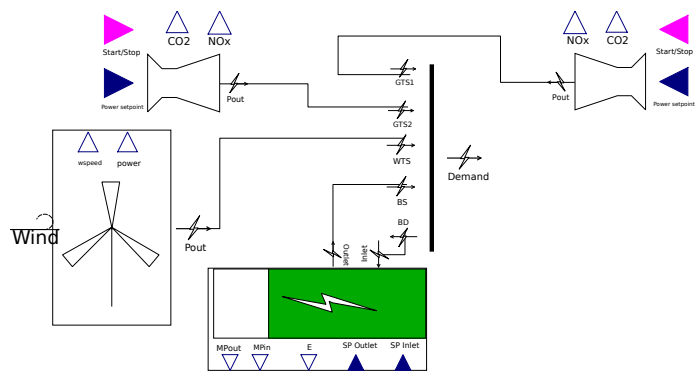

Figure 2. Physical Components Overview

The "Model" block from Figure 1 consists of the power components shown in Figure 2. The model includes two gas turbines, a wind power system configurable with zero or several wind turbines (no wake simulation yet), an energy storage system analogous to a battery, and a micro grid for energy balance. The model requires a wind source, a power demand, and set points to control the gas turbines and the energy storage system. These components are explained in the following sections.

\subsection{Wind Source and Power}

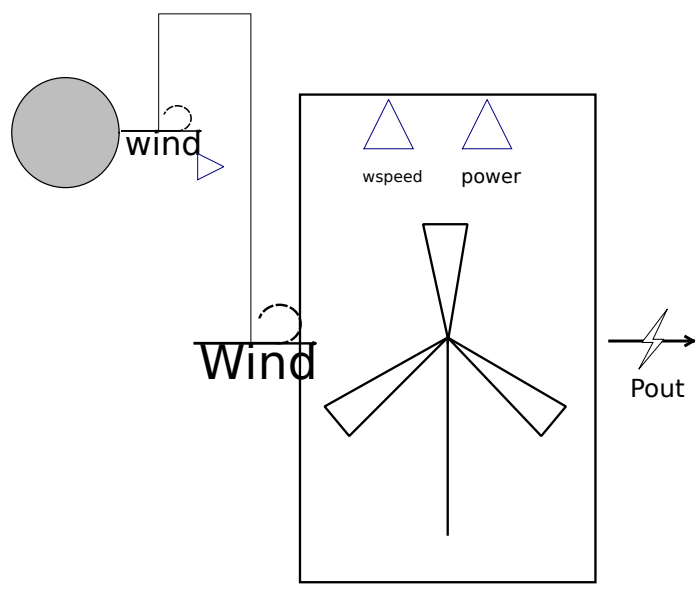

Figure 3. Wind Turbine Model

The wind turbine model in Figure 3 is purely data driven. It relates wind speed to generated power. This submodel also includes a configurable sensor model with the possibility to introduce errors in both the measured wind speed and the measured power. In this paper we rely on the
10 MW DTU wind turbine (Bak et al. 2013), and a wind speed dataset from ERA5 (ERA5 2020). The dynamic of the wind turbine system is shown in Figure 4 for a period of 7 days.
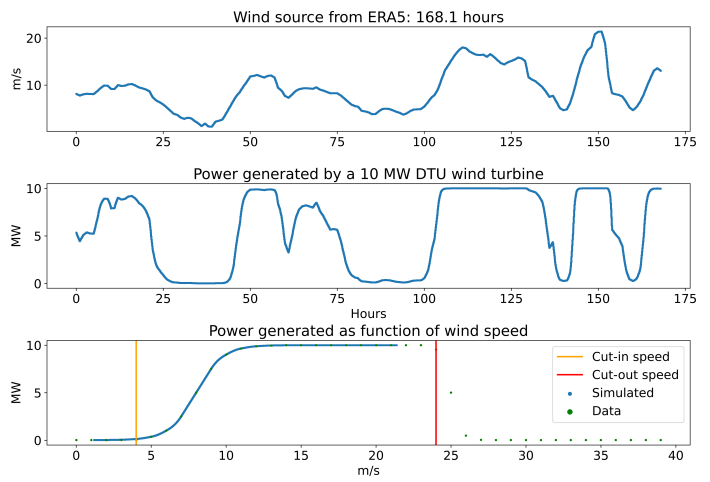

Figure 4. Wind Turbine Dynamic

\subsection{Storage System}

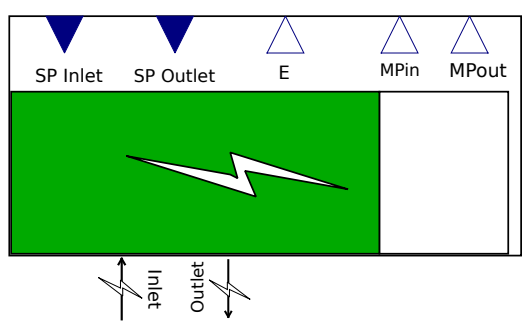

Figure 5. Energy Storage Model (Battery)

The storage model acts as a chargeable battery as shown in Figure 5. The model relates the charging power "SP Inlet" and the discharge power "SP Outlet" to the energy stored in the battery given as a \% of a maximum energy. The outputs are in the physical domain (power) which will be connected to a power grid. (see Section Grid). An illustration of the dynamic of the storage system is shown in Figure 6. The initial charge is set to $30 \%$. In this illustration, the battery starts supplying power when its level reaches $60 \%$ (signal to turn off gas turbines) and starts charging when its level is below $30 \%$ and the gas turbines have started (signal to turn on the gas turbines).
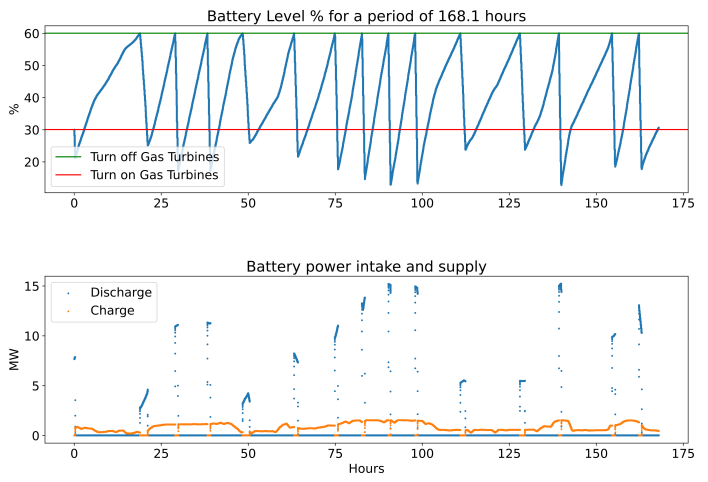

Figure 6. Illustration of the battery dynamic 


\subsection{Gas Turbine}

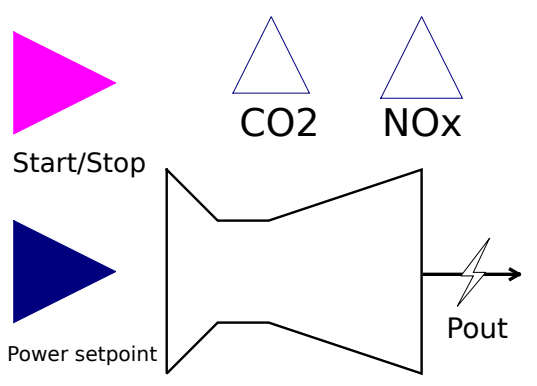

Figure 7. Gas Turbine Model

The model shown in Figure 7 simulates the dynamic of a gas turbine in a very simple form compared to more detailed models such as (Aguilera et al. 2019) and (Pires et al. 2018). The model receives two signals a start/stop signal and a power set point. The generated power in the physical domain is driven by a configurable startup duration and a ramp up controller. The model also outputs $\mathrm{CO} 2$ and NOx emission rates $(\mathrm{kg} / \mathrm{s})$ according to function relating power to emission curves shown in Figure 8. The plot shows the rates at which emission gases are released for two gas turbines operating between 5 and $8 \mathrm{MW}$ being the operational range used in this paper.
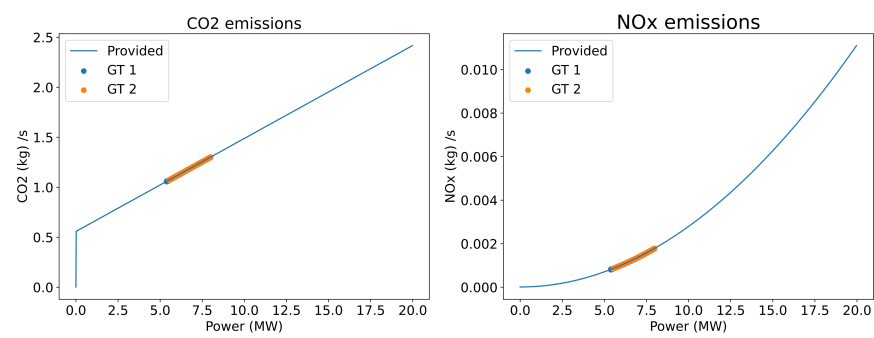

Figure 8. Gas Turbine Emission Curves

\subsection{Micro Grid Balance}

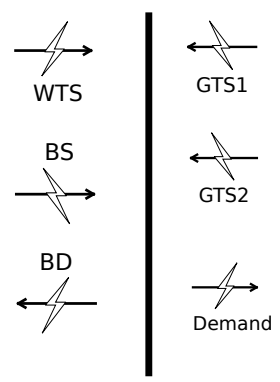

Figure 9. Micro Grid Model

The micro grid model in Figure 9 aims to keep the demand equal to the supply at any time during the simulation. This is achieved by allocating all the power surplus or deficit to a so-called "Losses" variable. An illustration of the grid dynamic is shown in Figure 10, with some minor power deficit at some time intervals (near 83 hours for example). Note that power deficits and losses are also indicators of the quality of the control system, which in this case suggests improvement. Nevertheless, the model remains consistent as the energy is kept balanced at the grid level. From a simulation perspective the power losses and deficits can be used as indicators for the microgrid stability. These could be further exploited to extent the simulation capabilities towards the electrical domain by relating power disbalance to frequency drop and gain as addressed in (Zografos, Ghandhari, and Eriksson 2018).
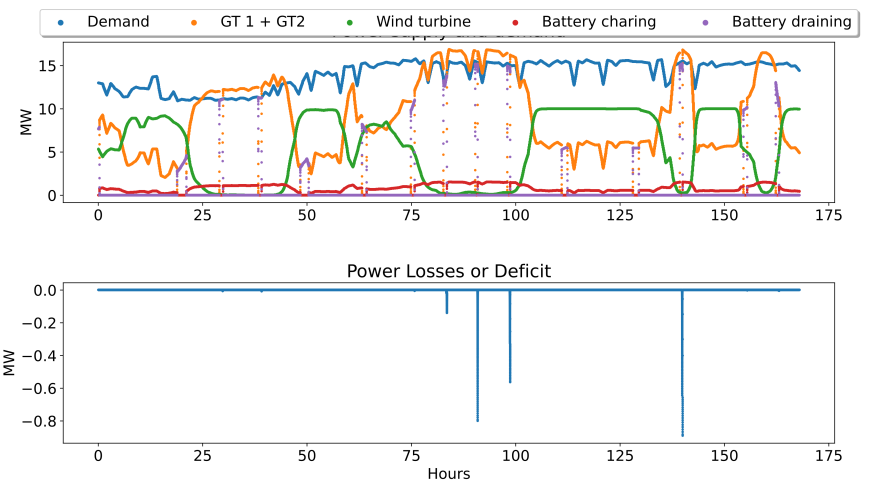

Figure 10. Illustration of the grid dynamic 


\section{Simulation Cases}

We vary the number the of wind turbines between 0 and 4 and plot the power supplied or consumed by each component, the oil platform power demand, and the amount of gas emissions. The parameters used to run the simulations are summarized in Table 1.

\subsection{Parameters}

Table 1. Sizes of compiler phases, lines of code.

\begin{tabular}{lr}
\hline Parameters & Values \\
\hline Trigger low limit & $30 \%$ \\
Trigger high limit & $60 \%$ \\
Battery max capacity & $22 \mathrm{MW}$ \\
Battery charging rate & 1 \\
Battery discharge rate & 1 \\
Battery Initial charge & $30 \%$ \\
Wind Turbines count & {$[1 \ldots 4]$} \\
Wind Turbine power & File \\
Gas Turbine max & $13 \mathrm{MW}$ \\
Gas Turbine startup time & $15 \mathrm{~min}$ \\
Time step & $1 \mathrm{~s}$ \\
Number of steps & $604,800 \mathrm{~s}$ (7 days) \\
\hline
\end{tabular}

Note. Assuming a floating structure of type semisubmersible such as the one defined in (Robertson et al. 2014) and a lithium ion battery system with an energy density of $163 \mathrm{Wh} / \mathrm{kg}$. By allocating $1 \%$ of the semisubmersible weight for the battery system, we roughly end up with a maximum energy of $22 \mathrm{MWh}$.

\subsection{Results and Interpretation}

The simulation without wind turbine in Figure 11 defines the base case, showing the situation as it is today. The whole power generation is provided by two gas turbines that are run synchronously. The simulation shows that 7 days of operations generate up to 1459 tonnes of $\mathrm{CO} 2$ mass, and 1.66 tonnes of NOx. In the scenario with only 1 wind turbine from Figure 12, we can notice that the battery charges and discharges frequently, because 1 wind turbine does not deliver sufficient power for the given demand, ending with 1085.99 tonnes of $\mathrm{CO} 2$ and 0.54 tonnes of NOx. With 2 wind turbines (Figure 13) on the other hand, we can already see that the battery stays at full charge for a relatively long periods, and even longer with 3 and 4 wind turbines (Figure 14 and Figure 15), with even fewer $\mathrm{CO} 2$ and NOx releases. However, the energy losses become important in the scenario with 4 wind turbines suggesting that the design of the system is oversized. Figure 16 and Figure 17 summarize the energy contribution from each component and the emitted masses of $\mathrm{CO} 2$ and $\mathrm{NOx}$.
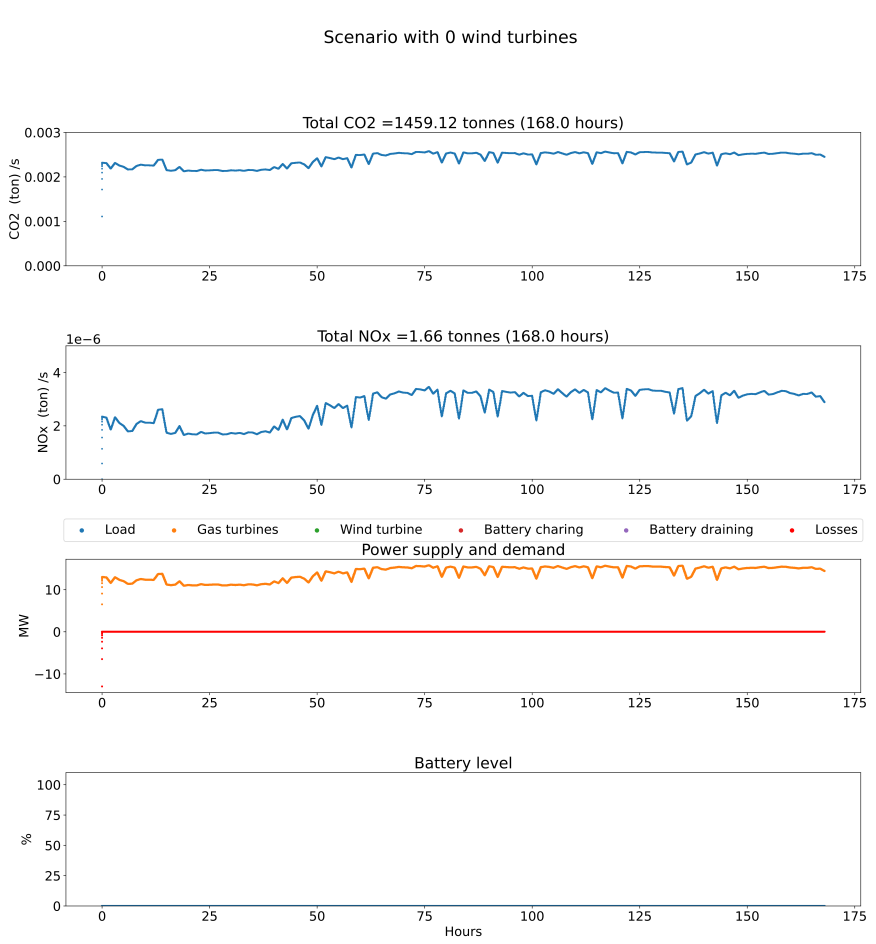

Figure 11. Base case without wind turbines and battery
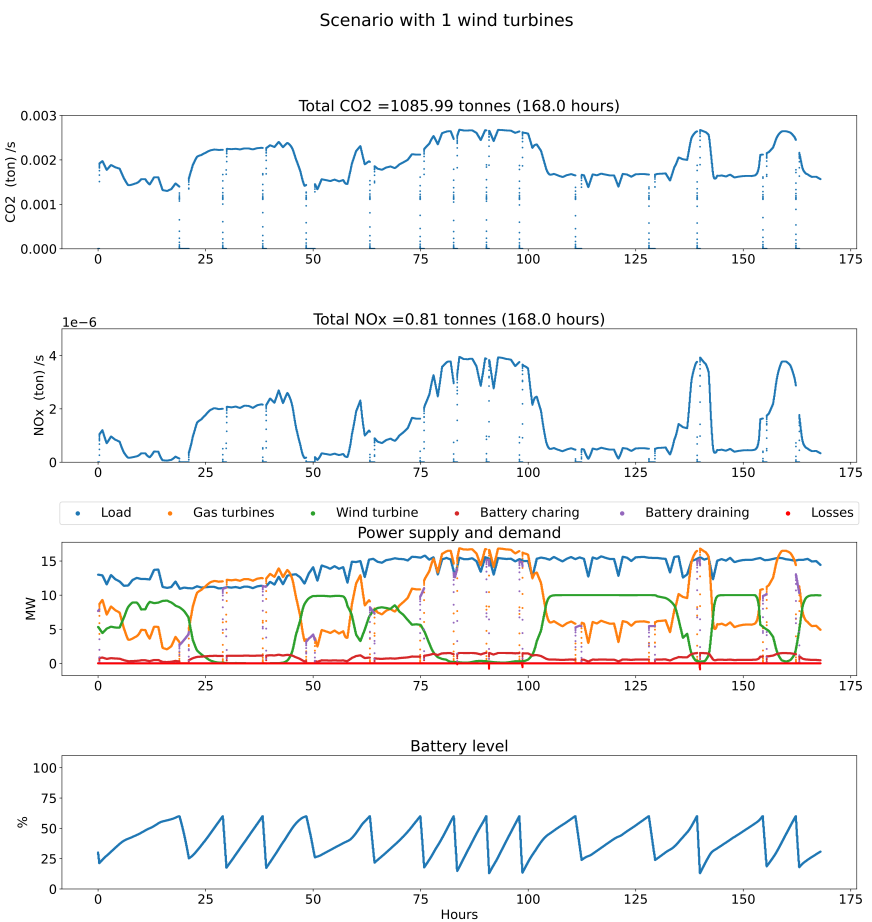

Figure 12. Simulation with 1 wind turbine 

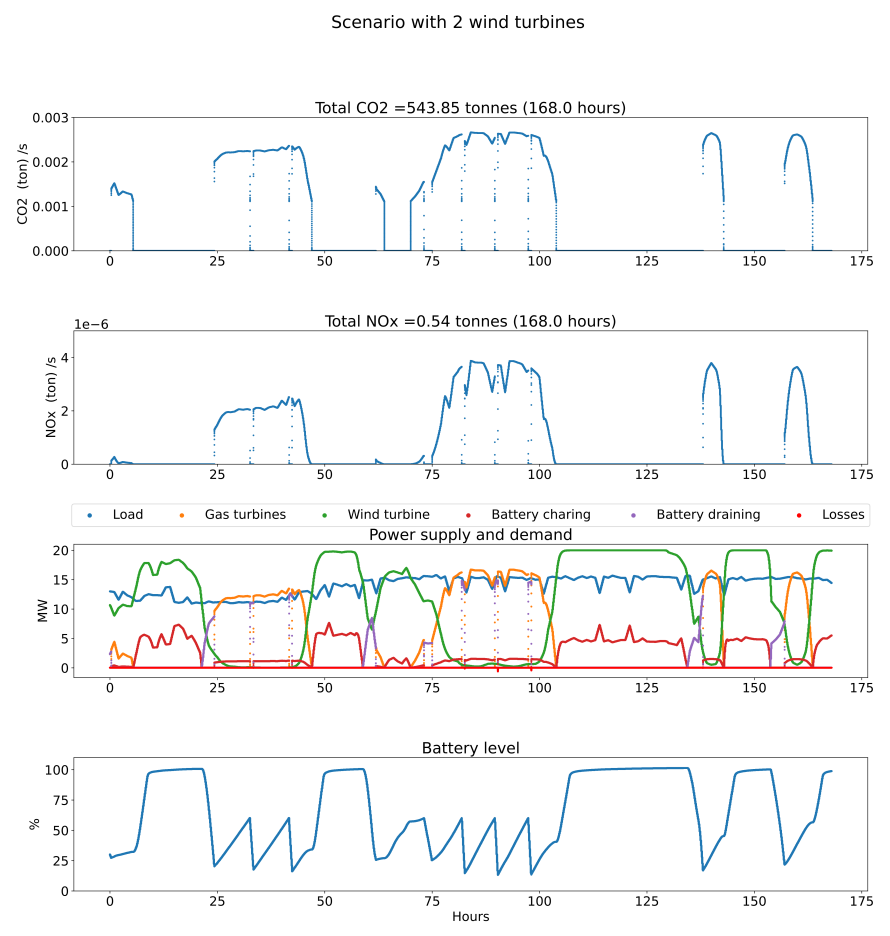

Figure 13. Simulation with 2 wind turbine
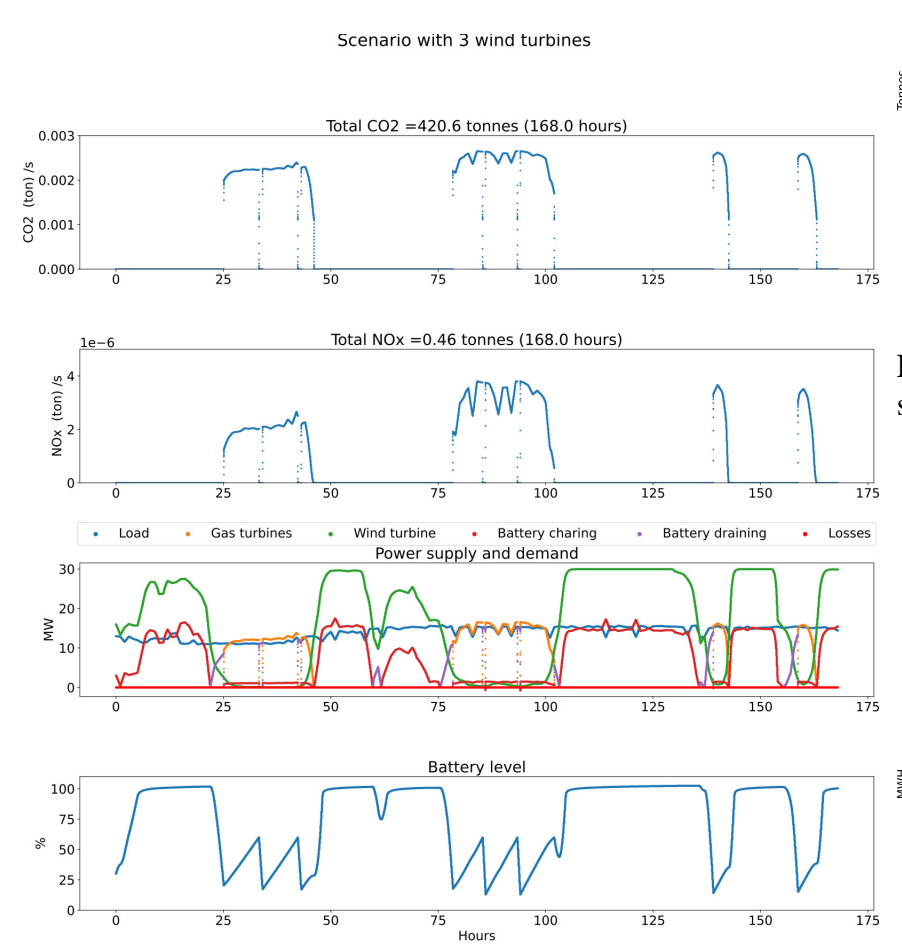

Figure 14. Simulation with 3 wind turbine
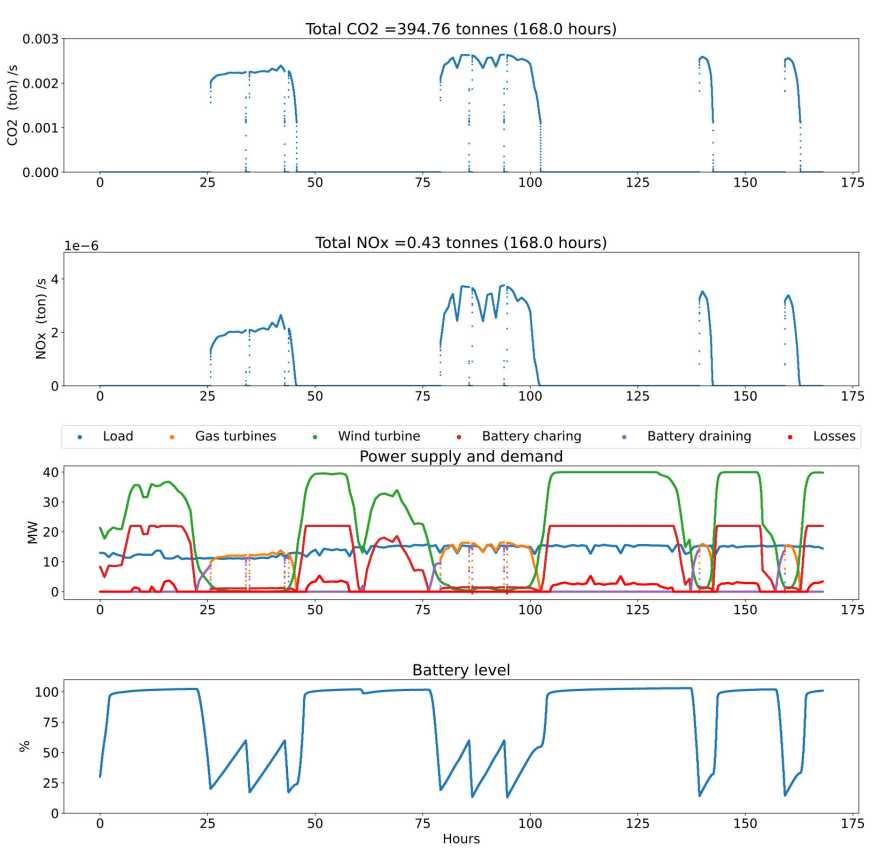

Figure 15. Simulation with 4 wind turbine
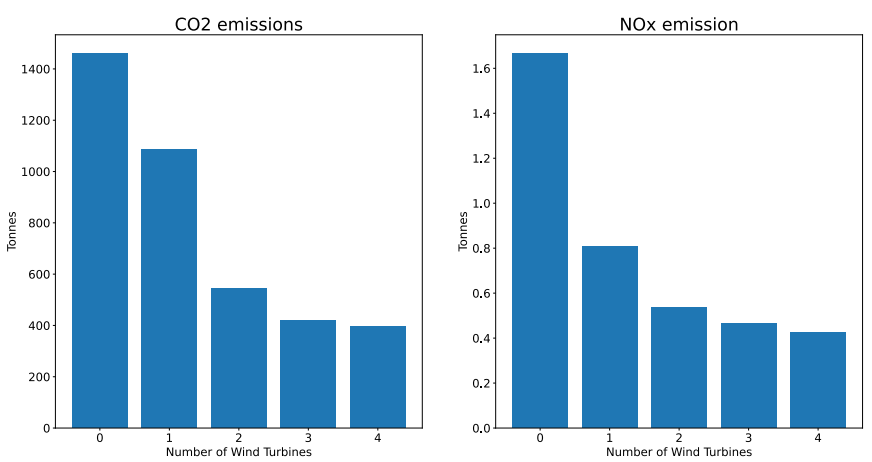

Figure 16. Summary of greenhouse gases emissions for 7 days simulation

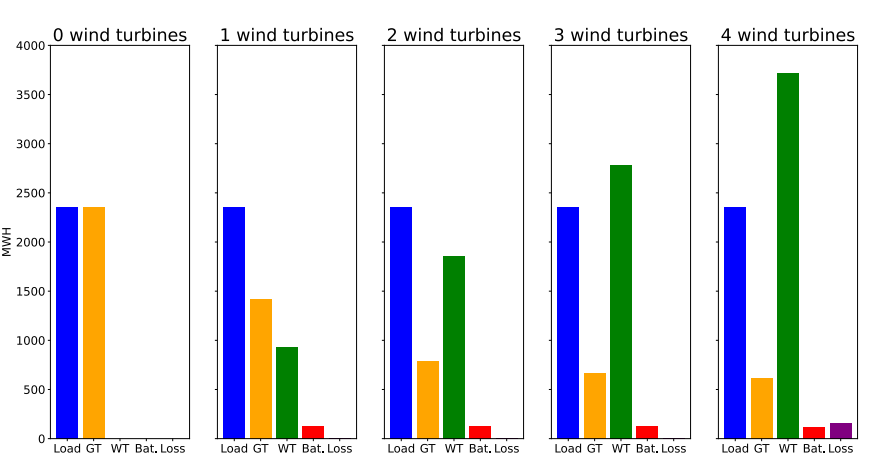

Figure 17. Power contribution for each scenario 


\section{Conclusions}

In conclusion, this paper has presented a simple and yet useful modelica model (ELOGOW 2021) for wind power integration with gas turbines and energy storages to reduce greenhouse emission gases for offshore oil and gas platforms. The paper also presented a possible control strategy to autonomously keep the micro grid in balance. Furthermore, in this model we deliberately avoided detailed component modeling as a design principle because these can vary between installation making model reconfiguration challenging and maybe even unpractical. However, many aspects of the model can be improved on literally every component presented. Here are some suggestions.

\subsection{Gas turbine model}

The amount of gas emissions depends on many parameters often unknown or are particular to every installation. Having good quality datasets on the gas emission would make it possible to have data driven models with better predictions. The same argument also holds for the startup and operational phases of gas turbines.

\subsection{Wind Turbines}

When more than one wind turbine is involved, their placement becomes important to avoid the wake effect, which in often cases is inevitable. Improving the wind turbine model with a wind speed weakening estimation would provide better estimations of the generated power. A possible approach would be to apply a fast wind park simulator such as (NREL 2021).

\subsection{Energy Storage System}

Energy storage systems have different characteristics. In this paper we model them as energy stored, power released, and power consumed. Yet, the dynamic of such systems is expected to be more complex and could eventually be derived from data (test cases) in combination with more detailed models such as (Gerl et al. 2014). The optimal size of the storage capacity will also be explored in future studies. Limiting factors are the physical size, weight, and cost of the components.

\subsection{Micro Grid}

The micro grid should be extended to the electrical domain and should be made more realistic for operations by adding frequency drop and rise as a function of the imbalance between the power supply and demand.

\subsection{Demand}

We have considered one dataset representing the power demand of a typical oil platform in the North Sea. However, the demand can vary in many ways not considered in this paper. Having different datasets or eventually demand simulating models would improve the quality of the estimations.

\subsection{Optimization}

The control strategy presented in this paper should be formalized as a multi objective optimization problem. These objectives could be reduced greenhouse gas emissions, improved battery life cycle, dynamic triggering limits, improved gas turbines usage, etc.

\section{Acknowledgements}

The authors would like to acknowledge Equinor of providing the datasets and IFE for advice on energy storage sizes. This work has been performed as part of the project Electrification of Oil and Gas Installation by Offshore Wind (308838 - KSPKOMPETANSE19) financed by The Research Council of Norway.

\section{References}

Aguilera, Miguel et al. (2019-02). "Coalesced Gas Turbine and Power System Modeling and Simulation using Modelica". In: DOI: $10.3384 /$ ecp1815493.

Bak, Christian et al. (2013). The DTU 10-MW Reference Wind Turbine. Danish Wind Power Research 2013 ; Conference date: 27-05-2013 Through 28-05-2013.

Bosch, Jonathan, Iain Staffell, and Adam D Hawkes (2018). "Temporally explicit and spatially resolved global offshore wind energy potentials". In: Energy 163, pp. 766-781. ISSN: 0360-5442. DOI: https://doi.org/10.1016/j.energy.2018.08. 153. URL: http://www.sciencedirect.com/science/article/pii/ S036054421831689X.

ELOGOW (2021). https://github.com/NORCEEnergy/ELOGOW.

ERA5 (2020). www.ecmwf.int/en/forecasts/datasets/reanalysisdatasets/era5.

Gerl, Johannes et al. (2014-03). “A Modelica Based Lithium Ion Battery Model”. In: DOI: 10.3384/ecp14096335.

He, Wei et al. (2010). "The Potential of Integrating Wind Power with Offshore Oil and Gas Platforms". In: Wind Engineering 34 (2), pp. 125-137. DOI: 10.1260/0309-524X.34.2.125. URL: https://doi.org/10.1260/0309-524X.34.2.125.

NREL (2021). https://github.com/NREL/floris.

Pires, Thiago S. et al. (2018-04). "Application of nonlinear multivariable model predictive control to transient operation of a gas turbine and NOX emissions reduction”. In: Energy 149. ISSN: 03605442. DOI: 10.1016/j.energy.2018.02.042.

Robertson, A. et al. (2014-09). "Definition of the Semisubmersible Floating System for Phase II of OC4”. In: DOI: 10. 2172/1155123. URL: https://www.osti.gov/biblio/1155123.

SSB (2020-11). https://www.ssb.no/en/natur-ogmiljo/statistikker/klimagassn.

Zografos, Dimitrios, Mehrdad Ghandhari, and Robert Eriksson (2018-08). "Power system inertia estimation: Utilization of frequency and voltage response after a disturbance". In: Electric Power Systems Research 161. ISSN: 03787796. DOI: 10. 1016/j.epsr.2018.04.008. 